\title{
Epistemologia da neuropsicologia: Fundamentos científicos da relação entre cérebro e comportamento
}

\author{
Epistemology of neuropsychology: Scientific foundations of the relationship \\ between brain and behavior
}

Davi Sidnei de Lima ${ }^{[a]}$, Tatiana Izabele Jaworski de Sá Riechi[b]

\footnotetext{
${ }^{[a]}$ Mestrado em Psicologia na Universidade Federal do Paraná (UFPR), Curitiba, PR - Brasil, e-mail: davisidneilima@hotmail.com

${ }^{[b]}$ Doutora em Ciências Médicas pela Universidade Estadual de Campinas (Unicamp), professora do Departamento de Psicologia e do Programa de Mestrado em Psicologia da Universidade Federal do Paraná Curitiba, PR - Brasil, e-mail: tatiriechi@hotmail.com
}

Recebido: 28/09/2011 Received: 09/28/2011

Aprovado: 04/04/2012 Approved: 04/04/2012

\begin{abstract}
Resumo
O advento das neurociências possibilitou a criação de modelos de interpretação das atividades mentais, e os conhecimentos alcançados com o auxílio das novas tecnologias têm redefinido conceitos e concepções sobre o ser humano. Nesse contexto, o estudo das relações entre o substrato neurológico e as funções psicológicas superiores é objeto da neuropsicologia. O desenvolvimento teórico e prático da neuropsicologia é ensejo para uma abordagem epistemológica, tendo em vista os diferentes modelos científicos construídos. Na apresentação dos fundamentos científicos da neuropsicologia será definida a noção de ciência, a reflexão epistemológica em torno do desenvolvimento das ciências e, a partir da perspectiva histórica, a constituição da neuropsicologia como área cientifica e as demandas contemporâneas dos novos aparatos tecnológicos. A reflexão epistemológica promove o aprofundamento do pensamento neuropsicológico, despertando para a necessidade de precisão conceitual e de medidas, formação de plataformas de dados neuropsicológicos e adoção das novas tecnologias.
\end{abstract}

Palavras-chave: Neuropsicologia. Epistemologia da neuropsicologia. História da neurociência.

\begin{abstract}
The advent of neuroscience has enabled the creation of models of interpretation of mental activities and knowledge achieved with the help of new technologies have redefined he concepts and ideas about being human. In this context, the study of relations between the neurological substrate and the higher psychological functions is the object of neuropsychology. The development of theoretical and practical opportunity to neuropsychology is an epistemological approach, in view of the different scientific models built. In the presentation of the scientific foundations of this system will be defined the notion of science, epistemological reflection about the development of science and from the historical perspective, the constitution of neuropsychology as a scientific area and the contemporary demands of new technological devices. The epistemological reflection promotes the deepening of thought neuropsychological awakening to the need for accuracy and conceptual measures, training of neuropsychological data platforms and adoption of new technologies.
\end{abstract}

Keywords: Neuropsychology. Neuropsychology epistemology. History of neuroscience.

Psicol. Argum. 2013 jul./set., 31 (74), 495-505 


\section{Introdução}

Aristóteles (1977) afirmou no inicio da obra A Metafísica a relação do conhecimento com a capacidade de espantar-se diante do mundo, sendo a ciência, enquanto conhecimento baseado na observação e na experiência, um fenômeno humano de assombro, o qual investiga a natureza e o próprio homem, proporcionando independência diante das forças da natureza. A forma de observar a natureza foi diversa ao longo dos séculos; os objetos dessa observação e o estudo sobre o cérebro acompanharam esse avanço, recebendo ao longo da história diferentes ênfases de acordo com as condições de conhecimento de cada época. Mas foi por meio das neurociências que isso se tornou um desafio para a tecnologia e o espírito humano (Churchland, 2004).

0 florescimento das neurociências teve inicio no século passado e a década de 1990 foi eleita como a década do cérebro (Gazzaniga, Ivry \& Mangun, 2006; Mader, 1996). O aprimoramento da tecnologia permitiu acessos que pareciam impossíveis. 0 cérebro, enquanto objeto de estudo, deixou de ser abordado de forma teórica ou a partir de pacientes com lesões cerebrais ou após a morte dos sujeitos, pois o imageamento permitiu analisar o tecido neural enquanto desenvolve suas funções (Beaumont, 2008). Os avanços das neurociências e a interface com a psicologia, por meio da neuropsicologia, provocam uma fundamentação epistemológica desse modo de fazer ciência. Assim, este ensaio teórico tem o objetivo de sintetizar os aspectos científicos da neuropsicologia: constituição histórica como ciência, a definição do seu objeto, os diferentes métodos de investigação, a construção de teorias e conceitos e a relação com a psicologia. Esse percurso exige indagar o que é ciência (1), a epistemologia enquanto reflexão crítica da ciência (2), a psicologia como área científica (3), as neurociências como campo de estudo da neuropsicologia e a constituição histórica do seu objeto de estudo (4).

\section{Ciência: atividade humana}

A ciência nasce da necessidade de explicar a realidade por meio do controle e da sistematização, caracterizando a busca por um método que permita a objetividade e a eliminação de interferências (Araújo, 1998; Nagel, 1968). Este ideal de explicação implica o desenvolvimento da ciência e, consequentemente, a construção de leis e teorias (Chauí, 2003; Ferrari, 1974; Japiassu, 1977).

O século XVII marca o inicio da ciência moderna (Andery, 2004), o empirismo ganha força e o conhecimento por meio da observação e da experimentação torna-se a base da ciência. Descartes (1596-1650) foi o principal estudioso dessa nova forma de fazer ciência. Em meados do século XIX, o positivismo impregna o pensamento filosófico europeu, fundamentado por Auguste Comte (1798-1857). Seu empreendimento consistiu em uma pesquisa sistemática do conhecimento humano e o controle dessa tarefa foi determinado por meio do método científico (Schultz \& Schultz, 2007). A ciência mantém o ideal da experimentação, aceitando somente o conhecimento objetivo, sistemático, universal e baseado no método hipotético-indutivo, o qual permite a construção de leis e a generalização a partir de teorias (Andery, 2004; Araújo, 1998; Chauí, 2003; Japiassu, 1977).

A palavra grega "método" é formada por duas partes e significa através/por meio (me-ta) do caminho (odos), e está relacionada à sistematização de procedimentos, comuns a várias ciências, que conduzem a obtenção das explicações, descrições e compreensões (Araújo, 1998; Ferrari, 1974). 0 método confere objetividade á abordagem do evento, definindo-o como objeto científico capaz de ser controlável, verificável, interpretável e possível de ser corroborado ou corrigido em outras elaborações (Andery, 2004; Chauí, 2003; Marconi \& Lakatos, 2010). A demonstração e a prova dos resultados são possíveis por meio do rigor e da sistematização proporcionadas pelo método, isso fundamenta a validade do estudo. 0 fato estudado em particular é relacionado a um todo, sendo unificado em uma explicação racional e possibilitando estabelecer uma teoria científica (indução) (Araújo, 1998; Nagel, 1968; Ruiz, 1979). A dimensão assumida pelo método supera as técnicas usadas, sendo o aparato tecnológico resultado dos obstáculos percebidos na realidade, apresentando indícios dos limites teóricos.

A especificidade do método científico depende dos contornos que assume, podendo ser: observação e descrição; experimentação; construção de sistemas formais e modelos explicativos; levantamento e teste de hipóteses, com explicações através de leis e/ou teorias. Assim, o método pode adquirir um caráter dedutivo, indutivo ou ambos, conduzindo a um conhecimento passível de validação, seja através de teste 
empírico, seja pelo confronto crítico de hipóteses e teorias. Importante considerar que a indução contém a marca de uma teoria aceita na prática científica; sendo assim, as ciências naturais não se fundamentam apenas no empirismo puro, possuindo também uma teoria que formata a formação de hipóteses (Abbagnano, 1970; Andery, 2004; Araújo, 1998; Chauí, 2003; Japiassu, 1977; Mason, 1980).

\section{Epistemologia}

0 método científico caracteriza as neurociências, pois o objeto material, o sistema nervoso central do ser humano, é bem determinado, assim como o método, conhecimento ob-tido a partir da observação e experimentação, tendo em vista conclusões objetivas, sistemáticas, capazes de definir conceitos, conduzir a leis e formar teorias, justificando-se, desse modo, a fundamentação epistemológica das neurociências (Almeida \& Gomes, 2001; Kristensen, Almeida \& Gomes, 2001; Marconi \& Lakatos, 2010; Rose, 1984).

Etimologicamente a palavra epistemologia remete ao "estudo da ciência" (epistheme = ciência, e logos = estudo, discurso) (Abbagnano, 1998), da sua investigação enquanto lógica do conhecimento. 0 vigor das ciências no século XIX exigiu da filosofia a abordagem do discurso científico, sendo a epistemologia uma disciplina filosófica que, por meio do método crítico, preocupa-se com a sintaxe e a semântica da ciência, bem como a crítica do conhecimento científico, sua história e desenvolvimento (Abbagnano, 1970; Araújo, 1998; Reale \& Antiseri 1990).

A ciência moderna fortaleceu o empirismo lógico, afirmando a capacidade do método indutivo em alcançar enunciados gerais a partir de operações lógicas. David Hume (1711-1776) questionou o indutivismo, considerando que não importa a quantidade de enunciados emitidos após inúmeras observações, pois jamais se alcançará um enunciado geral. 0 empirismo lógico argumentou a partir do cálculo de probabilidade de certeza das leis ou teorias científicas. Rudolf Carnap (1897-1970) propôs princípios lógicos para calcular tais proporções e o grau de probabilidade que elas conferem a uma teoria (Kneller, 1980).

Karl Popper (1902-1994) colocou-se contra o empirismo lógico, considerando que, apesar de toda probabilidade, uma teoria poderá sempre ser refutada pela prova que se apresentar a seguir (Popper, 1975). A falseabilidade, a possibilidade de negar os pressupostos de uma teoria, seria o melhor caminho para a sua fundamentação (Popper, 1986). Uma teoria que não pode ser refutada não é científica, é dogmática ou metafísica, sendo os principais exemplos o marxismo e a psicanálise (Popper, 1986). 0 verificacionismo e o refutacionismo são conditio sine qua non para a sobrevivência de uma teoria cientifica. Uma ciência elabora suas hipóteses para os problemas que se apresentam e as mantém até que sejam refutadas ou falsificadas por algum novo evento. Desse modo as teorias são verdades peremptórias, sobrevivem até serem contestadas ou até que não sejam capazes de explicar novos problemas (Chauí, 2003; Kneller, 1980; Popper, 1986). A validade de uma teoria está na abertura que deixa para fatos novos, os quais poderão falsificar os princípios e os conceitos que a fundamentam. A verossimilhança de uma teoria é apontada a partir das suas consequências lógicas (Popper, 1975).

Thomas Kuhn (1922-1996) construiu a reflexão epistemológica a partir da história da ciência e defendeu o progresso da atividade científica através das tradições de pesquisa. Isso porque elas possibilitam o desenvolvimento de uma ciência "madura" (Kuhn, 2003), caracterizada por uma sucessão de tradições de pesquisa, cada uma com teoria e métodos próprios, responsáveis por uma comunidade de cientistas durante certo tempo e sendo, finalmente, abandonada. Kuhn começou por chamar as ideias de uma tradição cientifica de "paradigma", designadas mais tarde por "matriz disciplinar", indicando, de modo geral, uma visão do mundo expressa numa teoria. 0 paradigma como um todo determina os problemas investigados, os dados considerados pertinentes, as técnicas de investigação usadas e os tipos de soluções admitidas. Kuhn divide a historia da ciência madura em fases "normais" e "revolucionárias". Durante a ciência normal, os pesquisadores desenvolvem as implicações de um paradigma ou matriz disciplinar o mais completamente possível, sendo um modo eficiente de solucionar problemas e ampliar uma teoria importante. As ciências "imaturas" (por exemplo, a Psicologia e a Sociologia) progridem pouco, pois carecem de paradigmas, divididas como estão em escolas rivais (Kneller, 1980; Kuhn, 1979; Kuhn, 2003).

Para Kuhn, todo paradigma prepara o caminho para o seu sucessor. Como qualquer teoria é uma abstração da realidade, nenhuma teoria pode pretender explicar todos os fenômenos em seu domínio. A pesquisa à sombra de um paradigma assegura que o maior número possível desses fatos será

Psicol. Argum. 2013 jul./set., 31 (74), 495-505 
encontrado o mais depressa possível. Quando um paradigma é amplamente elaborado e se defronta com as anomalias que assinalam os seus limites, os cientistas precisam procurar um sucessor, configurando uma revolução científica (Kuhn, 2003).

\section{Psicologia e cientificidade}

A constituição da Psicologia como ciência natural, diversa do estudo metafísico da filosofia, iniciou com a psicologia fisiológica de Wundt (1832-1920), realizada em laboratórios e enfatizando mais o método do que o objeto de estudo. A psicologia adotou o método experimental e centrou a investigação sobre o comportamento, encontrando a forma de constituir-se minimamente como ciência natural. A ampla divulgação do evolucionismo teve importância na definição do objeto da psicologia e o interesse pelas relações entre funções mentais superiores e correlato neural foi assumido por médicos, os quais estudavam as afasias e a relação com áreas cerebrais (Kristensen et al., 2001; Schultz \& Schultz, 2007).

Na primeira metade do século XX o funcionalismo orientou a pesquisa psicológica. Thorndike (18741949) considerava as discussões sobre mente e corpo uma questão ontológica da filosofia. Titchener (1867-1927), estruturalista, ficou entre o paralelismo, no qual a mente não depende do corpo para ser estudada, posição defendida por Wundt, e a concepção da experiência unitária entre mente e corpo. Watson (1878-1958) negou o problema ao descartar a consciência. Karl Spencer Lashley (1890-1958) investigou as implicações da localização das funções cerebrais (equipotencialidade); os psicólogos da Gestalt trabalharam conceitos de campo perceptual e isomorfismo e Donald Olding Hebb (1904-1985) desenvolveu a teoria de montagens neurais (Kristensen et al., 2001; Pinheiro, 2005).

\section{Neurociências}

As neurociências nascem do interesse crescente em torno do funcionamento do cérebro. No século XX acontecem várias descobertas a respeito das células neuronais e da estrutura cerebral. A unificação das ciências biológicas permitiu a fusão da ciência neural com a biologia celular e molecular, havendo ainda a relação entre o estudo do comportamento e a ciência do cérebro. A atual configuração do conhecimento sobre o sistema nervoso central (células neurais, cérebro e comportamento) foi possível a partir de cinco importantes disciplinas experimentais: anatomia, embriologia, fisiologia, farmacologia e psicologia (Fiori, 2008; Kandel \& Kupfermann, 1997; Kristensen et al., 2001).

No final do século XIX, o tecido neural tornou-se tema de uma ciência, quando os estudos do médico italiano Camillo Golgi (1843-1926) e do histologista espanhol Santiago Ramón y Cajal (1852-1934) descreveram os detalhes da estrutura das células neurais. O microscópio composto revelou a estrutura celular do tecido neural. Golgi desenvolveu um método de coloração por prata, que permitia a visualização das organelas celulares do neurônio, como corpo celular, dendritos e axônios. Cajal, através desse método, marcou células individuais demonstrando a composição do tecido neural por uma rede de células distintas; também desenvolveu conceitos que permitiram o inicio de uma doutrina do neurônio, seguindo o princípio de que os neurônios individuais são os elementos sinalizadores primários do sistema nervoso (Gazzaniga et al., 2006; Kandel \& Kupfermann, 1997).

A investigação fisiológica do sistema nervoso teve inicio no fim do século XVIII, com a descoberta das propriedades elétricas das células musculares e neurais, pelo médico e físico italiano Luigi Galvani (17371798). Os fisiologistas alemães Gustav Theodor Fritsch (1838-1907) e Eduard Hitzig (1838-1927) obtiveram de modelos animais a comprovação da relação entre estimulação do encéfalo e mudanças no comportamento. Essa descoberta levou os neuroanatomistas a desenvolver uma análise detalhada do córtex cerebral e da organização celular. Como essas regiões executavam diferentes funções, concluíram que deviam olhar de modo diverso o nível celular (Fiori, 2008; Gazzaniga et al., 2006; Kandel \& Kupfermann, 1997; Pinel, 2005).

No fim do século XIX a farmacologia ofereceu contribuição para a compreensão do sistema nervoso e do comportamento, comprovando que substâncias químicas interagem com receptores específicos nas células. As descobertas fundamentaram as pesquisas em torno da comunicação química das células neuronais (Kolb \& Whishaw, 2003).

\section{A neuropsicologia}

De acordo com estudos de alguns historiadores das neurociências, a neuropsicologia moderna 
começa com Donald Oding Hebb (1904-1985), Karl Spencer Lashley (1890-1958) e Aleksandr Romanovitch Luria (1902-1977). 0 termo neuropsicologia foi usado primeiramente em 1913, em uma preleção realizada por Sir William Osler, mas o desenvolvimento de fato iniciou nos anos 1940, na obra de Hebb, The Organization of Behavior: A Neuropsychological Theory (Engelhardt, Rozenthal \& Lacks, 1995; Kolb \& Whishaw, 2003). A neuropsicologia acompanhou os outros campos das neurociências, trazendo contribuições no que se refere à interação entre cognição, comportamento humano e atividade do sistema nervoso cerebral. A interdisciplinaridade mostra-se como pilar da neuropsicologia, visto a utilização de saberes da anatomia, fisiologia, neurologia, psiquiatria, psicologia e outras que contribuem para o avanço e mapeamento das funções cerebrais. $\mathrm{O}$ desenvolvimento histórico dos estudos da relação entre funções mentais, comportamento e sua base fisiológica ajudam a entender a constituição do objeto e do método científico da neuropsicologia (Kristensen et al., 2001; Lezak, 1995; Mader, 1996; Toni, Romanelli \& Salvo, 2005).

\section{Desenvolvimento histórico da neuropsicologia}

A investigação da relação do comportamento humano com bases biológicas tem raízes bastante remotas (Eccles, 1989; Landeira-Fernandez \& Castro, 2010; Pinheiro, 2005). A trepanação, prática de caráter terapêutico ou ritualista, que consistia na perfuração do crânio, de 7.000 a 20.000 anos atrás, deixou vestígios em vários continentes e denúncia a importância do cérebro para as curas ou ritos (Gordon, 1995). Durante a Idade Média a trepanação foi prática corrente, pois havia a busca pela "pedra da loucura" no cérebro de pacientes com sintomatologia psiquiátrica, e também a crença de que maus espíritos habitavam o cérebro desses pacientes, sendo possível sua retirada por meio de perfurações no crânio.

O papiro de Edwin Smith (Puigbó, 2002), descoberto no Egito em meados do século XIX, escrito por volta de 1700 a.C., apresenta considerações cirúrgicas sobre 48 casos, indicando a descrição clínica, os possíveis tratamentos e prognósticos, considerações sobre as meninges, líquor e medula espinhal, usando pela primeira vez o termo "encéfalo" (Bear, Connors \& Paradiso, 2002; Changeux, 1991; Zimmer, 2004). A Grécia realizou no século $V$ a.C. a distinção entre medicina e filosofia, assumindo uma perspectiva etiológica baseada na observação (Crivellato \& Ribatti, 2007). Mas a questão da relação entre corpo e alma não foi abandonada, sendo a saúde considerada uma justa harmonia dessas duas dimensões.

Pitágoras (580-510 a.C) delineia algumas observações localizacionistas ao defender a morada da mente no encéfalo e no coração a região da alma e das sensações; Alcmeon (500 a.C) apresentou uma descrição dos nervos ópticos e estudos sobre distúrbios funcionais do encéfalo, concluindo que nele estariam a inteligência e os sentidos; Hipócrates (460-370 a.C) estudou a epilepsia, considerando-a um distúrbio do encéfalo, localizando aí também a inteligência e as sensações (Reale \& Antiseri, 1990); Demócrito (470-360 a.C.) também fez a relação entre funções mentais e corpo, assim o pensamento teria sede no cérebro, a ira no coração e o desejo no fígado. Platão (428-347 a.C.) considerava o encéfalo como local de realização dos processos mentais, sendo a alma constituída por três partes bem definidas e com localizações diversas. 0 coração seria o local onde habitava a alma afetiva; no cérebro havia lugar para a alma intelectiva; e no ventre estaria localizada a alma concupiscível, relacionada ao apetite sexual. Aristóteles (384-322 a.C.) defendeu o coração como o centro das sensações, das paixões e do intelecto (tese cardiocentrista), sendo função térmica a atividade do encéfalo, necessária para refrigerar o corpo e a alma (Mondin, 1997; Pinel, 2005).

Os conhecimentos médicos de Galeno (203-130 a. C.), médico de gladiadores, tornaram-se dogmáticos por mais de mil anos; ele fazia analogias anatômicas sobre o funcionamento cerebral a partir da dissecação de animais. Galeno apresentava o cérebro humano formado pelo cerebrum, relacionado às sensações e memória, e o cerebellum, relacionado aos movimentos musculares. Os nervos eram dutos que conduziam os líquidos vitais ou humores, garantindo o registro das sensações e dos movimentos (Zimmer, 2004).

Os estudos de anatomia foram prejudicados pelo desenvolvimento da tradição cristã católica, a qual defendia a dignidade do ser humano e a sua não profanação pelos estudos invasivos da anatomia. Outros fatos como a invasão dos bárbaros que destruíram o Império Romano e a Biblioteca de Alexandria, colocaram a Europa numa época de insegurança e recolhimento, cabendo aos árabes preservar os documentos recuperados de Alexandria.

Psicol. Argum. 2013 jul./set., 31 (74), 495-505 
A renovação cultural renascentista, no início da Idade Moderna, contribuiu para inovações no campo da anatomia, destacando as figuras de Andreas Vesalius (1514-1564) e Leonardo da Vinci (14521519). Da Vinci obteve os moldes dos ventrículos cerebrais a partir da introjeção de cera líquida no cérebro. Isso evidenciou a existência de quatro ventrículos e não três como se acreditava. Os desenhos de Da Vinci ficaram na penumbra por aproximadamente 300 anos, período no qual fulgurou Vesalius com a publicação da obra De humani corporis fabrica libri septem (1543), na qual as ilustrações tiveram como base estudos realizados em cadáveres humanos (Mason, 1980; Pinheiro, 2005; Zimmer, 2004).

René Descartes (1596-1650) era conhecedor da obra de Vesalius, da descoberta da circulação do sangue por Harvey (1628) e aceitou o modelo mecânico vigente. 0 pensamento cartesiano contribuiu para o avanço das novas perspectivas da ciência na Idade Moderna, não só no que se refere às pesquisas físicas, mas também com relação às pesquisas biológicas e fisiológicas. Descartes (2009) recusou a noção aristotélica da alma como forma do corpo ou a alma como princípio vital proposta pelos escolásticos e apresentou a alma como substância pensante (res cogitans), de natureza diversa do corpo (res extensa), marcada pela indivisibilidade em contraste com o corpo divisível. 0 ponto de interação entre alma e corpo seria a glândula pineal, parte do cérebro não passível de duplicação (Brett, 1953; Damásio, 1996; Kristensen et al, 2001).

David Hartley (1705-1757), Albrecht von Haller (1707-1777) e Porchaska (1749-1820) continuaram as tentativas de abordarem a relação cérebro e mente no século XVIII; afirmações foram feitas a partir de estudos anatômicos sobre a sensação, percepção, memória e suas relações com o cérebro. Entretanto, o cérebro continuou sendo considerado uma massa homogênea, responsável por distribuir energia vital para o corpo. No século XIX a fisiologia dos sentidos tentou explicar o intelecto. Bell (1774-1842) diferenciou nervos sensórios e motores; Muller (18011858) apresentou a teoria das energias específicas dos nervos; Helmholtz (1821-1894) realizou estudos inovadores sobre ótica e audição; Weber (17951878) trabalhou com a sensação epitelial; Fechner (1801-1887) elaborou uma formula matemática capaz de medir o limiar sensorial (Kristensen et al, 2001; Schultz \& Schultz, 2007).

\section{Localizacionismo e Holismo: descrições a partir da experiência.}

As tentativas de relacionar as funções mentais e o comportamento conforme o método científico iniciaram no final do século XVIII, com o conhecimento sobre afasia (Kolb \& Whisaw, 2003). Franz Joseph Gall (1758-1828), anatomista, colocou a relação entre afasia e cérebro em primeiro plano, tornando-se um importante precursor da neuropsicologia. Gall, que seguia uma ontologia materialista, considerava o cérebro como o substrato essencial para o exercício das faculdades mentais, mostrando-se evidente para ele a relação entre lesão frontal e transtornos afásicos. Com Spurzheim (1776-1832) desenvolveu a teoria da relação entre protuberâncias na calota craniana e funções mentais, chamada de frenologia (frenos em grego = mente) (Pinel, 2005). Gall inaugurou a corrente localizacionista, afirmando que as funções mentais seriam clara e definitivamente localizáveis nas circunvoluções do córtex cerebral; contribui com a neuroanatomia e neurofisiologia, seu mérito foi revolucionar as neurociências com uma concepção não dualista, o que incentivou o início das neurocirurgias que tentavam alterar o comportamento. Tais experimentos indicam o nascimento da neurociência experimental (Herculano-Houzel, 2001; Kolb \& Whisaw, 2003; Toni et al., 2005).

Florens (1794-1867) combateu a frenologia através de experimentos com animais, relacionando o cerebelo com a motricidade, o bulbo com a respiração e o cérebro com a inteligência e a percepção; seguiu a perspectiva cartesiana e defendeu que o funcionamento da mente dependia da totalidade da massa cerebral. Suas ideias anteciparam a noção de equipotencialidade (plasticidade neuronal) e deram início ao movimento que resultou na corrente holista da função cerebral (Bear, Connors \& Paradiso, 2002; Kolb \& Whisaw, 2003; Pinheiro, 2005).

Bouillaud (1796-1881) foi defensor das ideias de Gall e procurou comprovar, a partir de estudos anatomoclínicos, a relação entre afasias e áreas do cérebro. Seu aluno, Pierre Paul Broca (1824-1880), médico e antropólogo, demonstrou a relação entre lobo frontal esquerdo e linguagem. As pesquisas de Broca, baseadas em avaliações clínicas e estudos anatômicos, são consideradas o inicio da neuropsicologia. Em 1865, Broca associou o hemisfério esquerdo com a produção da fala e com a ideia de dominância manual, pois lesões na porção do terceiro giro frontal do hemisfério esquerdo provocam 
prejuízo na articulação da fala, o que designou por afemia. Broca defendeu a assimetria funcional e dominância dos hemisférios (Gazzaniga et al., 2006; Kandel, 1997a; Toni et al., 2005).

Em 1874, Carl Wernicke (1848-1905) descreveu a relação causal entre a lesão no primeiro giro temporal esquerdo e uma das formas clínicas de afasia, a afasia sensorial: prejuízo na compreensão da linguagem. Wernicke descreveu ainda o que denominou de afasia de condução, na qual uma lesão afeta as fibras associativas que conectam o primeiro giro temporal ao terceiro giro frontal no hemisfério esquerdo, indicando a existência de um tipo de afasia na qual o paciente pode compreender a linguagem de outros e ter capacidade de produção, mas apresentar um distúrbio grave na repetição (Gazzaniga et al., 2006; Kandel, 1997a). Broca e Wernicke revitalizam o localizacionismo e outros autores, chamados de associacionistas, empenharam-se no estudo dos centros relacionados à linguagem. Funções não linguísticas também tiveram áreas cerebrais delimitadas. Em 1855, Panizza descreveu cegueira em pacientes com lesão na região occipital. No século XIX o caso de Phineas Gage foi relatado por John M. Harlow (1848-1849). Gage passou a apresentar alterações de comportamento após lesão no lobo frontal (Damásio, 1996; Kristensen et al., 2001). John Huhlings Jackson (1834-1911) imprimiu uma grande mudança nos estudos da relação entre cérebro e comportamento ao destacar a hierarquia funcional do sistema nervoso, lançando as bases para o futuro desenvolvimento da Teoria do Sistema Funcional de Vygotsky e Luria, cinquenta anos mais tarde (Toni et al., 2005).

\section{Estudos neuropsicológicos na Rússia}

Em paralelo ao pulsar de pesquisas neuropsicológicas na Europa, também a Rússia destacou-se com importantes estudos. Em 1789, L. Bolotov plantou as sementes das investigações e iniciou um intenso período de pesquisa sobre a afasia, sendo que a neurologia e a psicologia russa não apenas contribuíram com a descrição dos sintomas, como também explicaram os mecanismos psicofisiológicos causais inerentes aos transtornos da linguagem. A tradição fisiológica de Sechenov (1829-1905) e Ivan P. Pavlov (1849-1936) influenciou a investigação de Lev Vygotsky (1896-1934) na busca por alternativas às explicações localizacionistas e globalistas. Três princípios organizaram a posição sistêmica de Vygotsky quanto às funções corticais: a) interfuncionalidade; b) plasticidade; e c) modificabilidade. Essa orientação de pesquisa influenciou outros estudiosos como o neurologista Alexander Romanovich Luria (1902-1977), que inaugurou uma nova fase da neuropsicologia e propôs uma ciência que mantinha o equilíbrio com a fisiologia e a neurologia, sem perder a sua especificidade e estando imbricada com uma perspectiva humanista no que diz respeito às questões clínicas estudadas. Luria contribuiu para uma inovadora abordagem do exame clinico, pois influenciado pela sua ampla visão das funções corticais, passou a usar técnicas modestas, mas que fundamentaram uma nova metodologia de aproximação das manifestações comportamentais. A coerente compilação dos elementos envolvidos na relação entre cérebro e comportamento deu origem a um modelo teórico, até então inexistente, capaz de sustentar as investigações neuropsicológicas (Kristensen et al., 2001; Luria, 1981; Toni et al., 2005).

\section{A neurociência cognitiva}

O inicio da década de 1950 foi marcada nos Estados Unidos pela oposição ao behaviorismo, desenvolvendo-se a psicologia cognitiva e seu foco no processamento de informação (Witsken, Damato \& Hartlage, 2008). Os processos e representações mentais tiveram relevância para os trabalhos de George Miller em 1956, que investigou as limitações da capacidade do pensamento humano, tais como as da memória de curto-prazo. Muitos outros empreendimentos foram colocados em prática pela psicologia cognitiva, como a fundação de laboratórios e o questionamento das principais teses do behaviorismo. 0 conceito de um organismo moldado pelo ambiente foi alterado pelo médico neurologista Karl H. Pribram, o qual se posicionou favorável aos elementos de iniciativa, perspectivas e intenções dos organismos (Kristensen et al., 2001). A Inglaterra, a partir dos trabalhos da relação entre escrita e lesão cerebral de Marshall e Newcombe, em 1973, foi o palco do encontro entre a neuropsicologia e a psicologia cognitiva (Witsken et al., 2008). 0 paradigma do processamento da informação divulgado pela psicologia cognitiva logo foi usado para pesquisar também

Psicol. Argum. 2013 jul./set., 31 (74), 495-505 
outras funções cognitivas. A neuropsicologia cognitiva tornou-se uma disciplina mais coerente após o um conjunto de explicações sobre a cognição humana normal, desenvolvido por psicólogos cognitivos, principalmente em face da ênfase no desempenho de pacientes com lesão cerebral (Beaumont, 2008; Kristensen et al., 2001; Witsken et al., 2008).

\section{Discussão}

O avançar das ciências nos últimos decênios revelou, a partir do aprimoramento da aquisição do conhecimento, novas perspectivas sobre a realidade humana. Não apenas o mundo externo foi investigado, mas o homem foi explorado em toda a sua interioridade na busca de entendimento dos seus aspectos biológicos e, consequentemente, também dos elementos que o tornam um gênero distinto de todos os outros seres viventes, as suas funções mentais superiores (Boncinelli, 2005). Os avanços em várias áreas de pesquisa do sistema nervoso central, tais como fisiologia, psicofarmacologia, neurologia e psicologia, garantiram descobertas de diversos níveis de complexidade (Kandel, 1997b). A pura especulação da filosofia foi abandonada em favor da experimentação e o comportamento investigado em suas bases biológicas. A linguagem, o pensamento, a memória, a atenção, enfim, todas as chamadas faculdades mentais, passaram a ser observadas e o seu substrato cerebral dissecado (Gazzaniga et al., 2006, Kolb \& Whisaw, 2003; Kristensen et al., 2001; Toni et al., 2005). A pergunta sobre quem é o homem adquiriu diferentes contornos ao longo das transformações da investigação científica, sendo que uma das maiores revoluções advém da investigação sobre os aspectos neurológicos e comportamentais do ser humano, proporcionada pelas neurociências (Boncinelli, 2005).

\section{Interface epistemológica da neuropsicologia}

K. Popper e T. Kuhn em suas epistemologias, apesar de genéricas e com considerações reticentes quanto à psicologia, fornecem categorias e conceitos passíveis de serem aplicados na reflexão dos fundamentos científicos da neuropsicologia. Popper argumenta que a validade de uma teoria não depende da afirmação dos seus pressupostos por meio da experiência, mas sim a tentativa de falsificá-la, tentando encontrar elementos que não corroborem as suas conclusões. Uma teoria não corroborada dá espaço para outra teoria que responde aos problemas da antiga e aos novos desafios. Popper defende o progresso da ciência a partir da constante substituição das teorias, as quais avançam em suas respostas aos problemas. Seguindo essa lógica, uma teoria é herdeira da outra, isso pelo contato de conceitos e contribuições recebidas da teoria anterior.

Kuhn considerou equivocada essa noção de evolução das ciências, pois para ele uma teoria é diversa da outra. Os conceitos de uma teoria não podem ser utilizados em outra, tendo em vista que os problemas, as condições e vários fatores científicos são diversos. Kuhn defende que o progresso das ciências se dá por meio de revoluções científicas, pois uma teoria que não responde mais aos problemas que aparecem é substituída por outra capaz de responder às questões da teoria anterior e aos novos problemas que apareceram. Para ele, a nova teoria não utiliza os conceitos da anterior da mesma forma, ela é inovadora. Surge, assim, a ciência normal, a qual corresponde a um paradigma que dá sustentação às varias teorias respondentes dos problemas de uma época (Chauí, 2003; Kneller, 1980; Kuhn, 1979; Popper, 1986).

O diálogo entre Popper e Kuhn pode ser estimulado para análise da história da formação das neurociências e constituição da teoria neuropsicológica. Seguindo o raciocínio de Popper, percebem-se as conjecturas em torno da relação entre funções mentais e sistema biológico, estabelecidas ao longo da reflexão sobre esse tema; aconteceram avanços especulativos até a adoção do método experimental. No desenvolvimento dessas explicações os conceitos foram sendo passados de teoria para teoria e, conforme apresenta Kuhn, não foram usados da mesma forma nos diferentes sistemas teóricos, ocasionando desentendimentos e equívocos. Gall e Florens assumiram posições teóricas diversas, sendo que Florens pesquisou a posição localizacionista de Gall e manifestou as inconsistências, realizando a falseabilidade da frenologia, conforme proposto por Popper. A frenologia não se sustentou e por isso foi substituída por novas posições localizacionistas, com conceitos herdados da frenologia, mas com novas configurações.

Kuhn estabelece as revoluções cientificas a partir das mudanças de paradigma. E o surgimento 
recente da neuropsicologia, com sua fundamentação do ser humano como um todo biopsicosocial, constitui uma revolução científica, principalmente por adotar uma visão nova para uma problemática velha, por expandir os sistemas de problemas, por obter êxito onde enfoques alternativos fracassam e por promover a fusão de disciplinas anteriormente separadas (Riechi, 1996).

Bilder (2011) propõe uma agenda para a Neuropsicologia contemporânea, sendo uma de suas tarefas fundamentais a formalização de conceitos e medidas de maneira ampla e bem definida, capaz de indicar com precisão o que a neuropsicologia aborda, além de agregar o conhecimento produzido por outras áreas neurocientíficas de interface. As transformações científicas provocadas pelas novas tecnologias exigem a clareza de conceitos e a definição de paradigmas no interior da neuropsicologia. Outra meta seria a construção de redes colaborativas de troca de conhecimentos, pois as tecnologias da informação permitem a elaboração de plataformas de dados, capazes de servir de base para os mais diversos centros de pesquisa. Bilder (2011) também sugere a incorporação das novas tecnologias nas práticas neuropsicológicas. Assim, a avaliação neuropsicológica seria beneficiada pela utilização de técnicas computacionais, o que poderia trazer maior precisão na aplicação de testes.

\section{Problemas}

Uma teoria científica, tanto para Kuhn quanto para Popper, tem seu valor de verdade provado pelos problemas que suscita, pois provocam as pesquisas e o desenvolvimento teórico. A agenda proposta por Bilder para a Neuropsicologia revela alguns dos problemas constitutivos dessa ciência contemporânea, sendo que outros dois problemas fundamentais também estão no bojo da pesquisa neuropsicológica, sendo que a solução dada é determinante para a construção do conhecimento. Esses problemas são a questão do método e a discussão entre monismo e dualismo. Quanto ao método, o maior problema refere-se à produção de conhecimento baseada amplamente em inferências realizadas após o funcionamento do cérebro, ou seja, não é um conhecimento produzido diante do cérebro em funcionamento. As novas técnicas de imageamento cerebral estão superando alguns elementos deste entrave, mas ainda lentamente (Beaumont, 2008). 0 debate sobre as bases biológicas do comportamento sempre esbarra no histórico conflito das relações entre mente e corpo, estabelecendo-se duas concepções básicas, o dualismo e o monismo. 0 dualismo defende a existência de dois princípios irredutíveis e o monismo reduz o conjunto das coisas à unidade, tanto do ponto de vista da substância quanto no que se refere às leis lógicas ou físicas (Damásio, 1996; Pinheiro, 2005).

\section{Considerações finais}

A ciência se desenvolve na sociedade e responde aos desafios de cada época. Um trabalho científico também reflete em sua natureza, metodologia e terminologia os interesses externos às pesquisas, mesmo os propósitos e preconceitos do próprio cientista (Rose, 1984). A neuropsicologia historicamente testemunha os condicionamentos impostos pelos métodos de acesso às funções mentais e produz conhecimento com consequências científicas e sociais, sendo necessário abordar a sua importância para o conhecimento psicológico e para a sociedade.

Cabe considerar que a ciência psicológica não se reduz à neuropsicologia, sendo esta um ramo científico que apresenta horizontes amplos para a psicologia, possibilitando a integração de compreensões, bases conceituais e muitas questões simbólicas (Kristensen et al., 2001). A revista Nature (Anônimo, 2009, oct. 15) alertou para as contribuições das neurociências à psicologia científica, pois durante os últimos 20 anos, a ciência tem feito grandes progressos em direções que poderiam apoiar a psicologia clínica, tais como a neuroimagem, assim como a genética molecular e comportamental e neurociência cognitiva. Desse modo, a interação da psicologia com a neuropsicologia afirma o caráter interdisciplinar desta e, atualmente, contribui para o conhecimento do cérebro e suas relações com o comportamento através da neuropsicologia clínica e da reabilitação cognitiva.

0 pensamento humano continua a ser um desafio para a ciência. E a disciplina neuropsicológica mostra-se capaz de investigar cientificamente este desafio e oferecer respostas. Contudo, há de se considerar com parcimônia toda promessa científica. Considerar esse fato irrelevante é correr o risco de gerar falsas esperanças e criar celeumas, tendo em

Psicol. Argum. 2013 jul./set., 31 (74), 495-505 
vista os abusos que podem ser gerados, tais como já testemunhados pela história científica (Fiori, 2008).

Aspectos de base da ciência estabelecem a neuropsicologia como disciplina científica interdisciplinar e as raízes remotas do seu objeto de investigação fundamentaram as mais diversas teorias, mas com o desenvolvimento das neurociências houve novas consequências para as descobertas das relações mentais com o sistema biológico. Contudo, essa investigação continua em aberto (Bennett \& Hacker, 2003; Churchland, 1996; Churchland, 2004) e suscitando o espírito científico, exigindo constantemente uma reflexão epistemológica, pois as consequências da posição tomada irão refletir na visão de homem e no modo de fazer ciência.

\section{Referências}

Abbagnano, N. (1970). História da Filosofia. (Vol. 1). Lisboa: Editorial Presença.

Abbagnano, N. (1998). Dicionário de filosofia. São Paulo: Martins Fontes.

Andery, M. A. M. (2004). Para compreender a ciência. São Paulo: Educ.

Anônimo (2009, oct. 15). Psychology: A reality check. Nature, 461(7266).

Araújo, I. L. (1998). Introdução à Filosofia da Ciência (2. ed.). Curitiba: Editora da UFPR.

Aristóteles (1977). Metafísica. (Os Pensadores). São Paulo: Abril Cultural.

Bear, M. F., Connors, B. W., \& Paradiso, M. A. (2002). Neurociências: Desvendando o sistema nervoso. Porto Alegre: Artmed.

Beaumont, J. G. (2008). The discipline of neuropsychology. In J. G. Beaumont. Introduction to neuropsychology. (2nd ed., pp. 3-21). New York: Guilford.

Bennett, M. R., \& Hacker, P. M. S. (2003). Philosophical foundations of neuroscience. Oxford: Blackwell.

Bilder, R. (2011). Neuropsychology 3.0: Evidence-based science and practice. Journal of the International Neuropsychological Society, 17(1), 7-13.

Brett, G. S. (1953). A History of psychology. Londres: Allen \& Unwin.
Boncinelli, E. (2005). Necessità e contingenza della natura umana. Micromega, almanacco di filosofia, 4, 8-27.

Changeux, J. P. (1991). O homem neural. Lisboa: Dom Quixote.

Chauí, M. (2003). Convite à Filosofia. São Paulo: Ática.

Churchland, P. M. (2004). Matéria e consciência: Uma introdução contemporânea à filosofia da mente. São Paulo: Unesp.

Churchland, P. S. (1996). Neurophilosophy: Toward a unified science of the mind- brain. Cambridge: MIT Press.

Crivellato, E., \& Ribatti, D. (2007). Soul, mind, brain: Greek philosophy and the birth of neuroscience. Brain Research Bulletin, 71, 327-336.

Damásio, A. (1996). O Erro de Descartes. São Paulo: Companhia das Letras.

Descartes, R. (2009). Discurso do método. São Paulo: Martins Fontes.

Eccles, J. (1989). A Evolução do cérebro. A criação do Eu. Lisboa: Instituto Piaget.

Engelhardt, E., Rozenthal, M., \& Lacks, J. (1995). Neuropsicologia: História. Revista Brasileira de Neurologia, 31(2), 107-113.

Ferrari, A. T. (1974). Metodologia da ciência. (3. ed.). Rio de Janeiro: Kennedy.

Fiori, N. (2008). As Neurociências Cognitivas. Petrópolis: Vozes.

Gazzaniga, M. S., Ivry, R. B., \& Mangun, G. R. (2006). Neurociência Cognitiva. A Biologia da Mente. Porto Alegre: Artmed.

Gordon, R. (1995). A assustadora história da medicina. Rio de Janeiro: Ediouro.

Herculano-Houzel, S. (2001). A frenologia e o nascimento da neurociência experimental. In R. Lent. Cem bilhões de neurônio: Conceitos fundamentais de neurociência (pp. 20-21). São Paulo: Atheneu.

Japiassu, H. (1977). Introdução ao pensamento epistemológico. (2 ed.). Rio de Janeiro: Francisco Alves.

Kandel, E. R. (1997a). Cérebro e Comportamento. In E. R. Kandel, J. H. Schwartz \& T. M. Jessell (Org.). Fundamentos da neurociência e do comportamento. (pp. 5-15). Rio de Janeiro: Guanabara Koogan. 
Kandel, E. R. (1997b). Genes e comportamento. In E. R. Kandel, J. H. Schwartz, \& T. M. Jessell (Org.). Fundamentos da neurociência e do comportamento (pp. 445-460). Rio de Janeiro: Guanabara Koogan.

Kandel, E. R., \& Kupfermann, I. (1997). Das células nervosas à cognição. In E. R. Kandel, J. H. Schwartz, \& T. M. Jessell (Org.). Fundamentos da neurociência e do comportamento (pp. 261-280). Rio de Janeiro: Guanabara Koogan.

Kuhn, T. S. (1979). Reflections on my critics. In I. Lakatos \& A. Musgrave (Ed.). Criticism and the growth of knowledge (pp. 231-278). New York: Cambridge University Press.

Kuhn, T. S. (2003). A Estrutura das Revoluções científicas. São Paulo: Perspectiva.

Kneller, G. F. (1980). A ciência como atividade humana. São Paulo: Zahar.

Kolb, B., \& Whishaw, I. Q. (2003). The depelopment of neuropsychology. In B. Kolb \& I. Q. Whishaw. Fundamentals of human neuropsychology. (5th ed., pp. 1-26). New York: Worth Publishers.

Kristensen, C. H., Almeida, R. M. M., \& Gomes, W. B. (2001). Desenvolvimento histórico e fundamentos metodológicos da neuropsicologia cognitiva. Psicologia: Reflexão e Crítica, 14(2), 259-274.

Landeira-Fernandez, J., \& Castro, F. S. (2010). Alma, mente e cérebro na pré-história e nas primeiras civilizações humanas. Psicologia: Reflexão e Crítica, 23(1), 37-48.

Lezak, M. D. (1995). Neuropsychological assessment. (3rd ed.). New York: Oxford University Press.

Luria, A. R. (1981). Fundamentos de neuropsicologia. Rio de Janeiro: LTC; São Paulo: Edusp.

Mader, M. J. (1996). Avaliação neuropsicológica: Aspectos históricos e situação atual. Psicologia: Ciência $e$ Profissão, 16(3), 12-18.

Marconi, M. A., \& Lakatos, E. M. (2010). Fundamentos de metodologia científica. São Paulo: Atlas.

Mason, L. F. (1980). História da ciência: As principais correntes do pensamento científico. Rio de Janeiro: Globo.

Mondin, B. (1997). Introdução à filosofia: Problemas, sistemas, autores e obras. São Paulo: Paulus.

Nagel, E. (1968). La estructura de la ciência: Problemas de la lógica de la investigación científica. Buenos Aires: Paidós.
Pinel, J. P. J. (2005). Biopsicologia. São Paulo: Artmed.

Pinheiro, M. (2005). Aspectos históricos da neuropsicologia: Subsídios para a formação de educadores. Educar em Revista, 25, 143-174.

Popper, K. (1986). Conjecturas e refutações. Brasília: Ed. UnB.

Popper, K. (1975). A lógica da pesquisa científica. São Paulo: Cultrix.

Puigbó, J. J. (2002). El papiro de Edwin Smith y la civilización egípcia. Gaceta Médica de Caracas, 110 (3), 378-385.

Reale, G., \& Antiseri, D. (1990). História da Filosofia (3 Vol.). São Paulo: Paulus.

Riechi, T. I. J. S. (1996). Uma proposta de leitura neuropsicológica dos problemas de aprendizagem. Dissertação de Mestrado em Educação não publicada, Universidade Federal do Paraná, Curitiba.

Rose, S. (1984). O cérebro consciente. São Paulo: Alfa-omega.

Ruiz, J. A. (1979). Metodologia científica: Guia para eficiência nos estudos. São Paulo: Atlas.

Schultz, D., \& Schultz, S. E. (2007). História da psicologia moderna. São Paulo: Thomson.

Toni, P. M., Romanelli, E. J., \& Salvo, C. G. (2005). A Evolução da neuropsicologia: Da anti-guidade aos tempos modernos. Psicologia Argumento, 23(41), 47-55.

Witsken, D. E., Damato, R. C., \& Hartlage, L. C. (2008). Understanding the past, present and future of clinical neuropsychology. In R. C. Damato \& L. C. Hartlage. Essencials of neuropsychological assessment, treatment, planning for rehabitation. (3rd ed., pp. 1-29). New York: Springer Publishing.

Zimmer, C. (2004). A Fantástica História do Cérebro: o funcionamento do cérebro humano. Rio de Janeiro: Elsevier.

Psicol. Argum. 2013 jul./set., 31 (74), 495-505 